Revue internationale P.M.E.

Économie et gestion de la petite et moyenne entreprise

\title{
Les petites entreprises en Pologne : les années récentes et celles de 1980
}

\section{Maciej H. Grabowski}

Volume 5, numéro 1, 1992

URI : https://id.erudit.org/iderudit/1008135ar

DOI : https://doi.org/10.7202/1008135ar

Aller au sommaire du numéro

\section{Éditeur(s)}

Presses de l'Université du Québec

ISSN

0776-5436 (imprimé)

1918-9699 (numérique)

Découvrir la revue

Citer cette note

Grabowski, M. H. (1992). Les petites entreprises en Pologne : les années récentes et celles de 1980. Revue internationale P.M.E., 5(1), 93-110. https://doi.org/10.7202/1008135ar
Résumé de l'article

Cette analyse montre comment s'est effectué le développement des PME en Pologne entre les années 1980 et 1990 à travers les changements intervenus dans l'économie polonaise, les principales politiques macro-économiques mises de l'avant et leur impact sur le développement des PME.

Dans un premier temps, l'auteur relève les changements légaux qui ont favorisé la croissance rapide des PME incorporées ainsi que les changements économiques intervenus à la fin des années 1980. Dans un deuxième temps, l'auteur présente les principes de base qui otn guidé ces changements (les éléments et résultats du plan Balcerowcicz), dresse le profil du comportement des entreprises privées face à ces changements et montre comment est survenue l'accélération du développement des PME dans la seconde moitié de 1990.

Enfin, l'auteur décrit l'impact des politiques macro-économiques polonaises sur le développement des PME : la fiscalité (opposée au développement des PME), les hauts taux d'intérêts bancaires, l'inflation provoquée de même que l'échec de la politique de taxation et de la politique fiscale.
Ce document est protégé par la loi sur le droit d'auteur. L'utilisation des services d’Érudit (y compris la reproduction) est assujettie à sa politique d'utilisation que vous pouvez consulter en ligne.

https://apropos.erudit.org/fr/usagers/politique-dutilisation/ 


\title{
Les petites entreprises en Pologne : les années récentes et celles de 1980
}

\author{
Maciej H. GRABOWSKI \\ Institut de Gdansk pour l'économie de marché*
}

\begin{abstract}
RÉSUMÉ
Cette analyse montre comment s'est effectué le développement des PME en Pologne entre les années 1980 et 1990 à travers les changements intervenus dans l'économie polonaise, les principales politiques macro-économiques mises de l'avant et leur impact sur le développement des PME.
\end{abstract}

Dans un premier temps, l'auteur relève les changements légaux qui ont favorisé la croissance rapide des PME incorporées ainsi que les changements économiques intervenus à la fin des années 1980. Dans un deuxième temps, l'auteur présente les principes de base qui otn guidé ces changements (les éléments et résultats du plan Balcerowcicz), dresse le profil du comportement des entreprises privées face à ces changements et montre comment est survenue l'accélération du développement des PME dans la seconde moitié de 1990.

Enfin, l'auteur décrit l'impact des politiques macro-économiques polonaises sur le développement des PME : la fiscalité (opposée au développement des PME), les hauts taux d'intérêts bancaires, l'inflation provoquée de même que l'échec de la politique de taxation et de la politique fiscale.

* Maciej H. Grawbowski a obtenu son diplôme à l'Université de Gdansk. Il est présentement directeur général adjoint à l'Institut pour l'économie de marché de Gdansk. Il se spécialise en recherche sur les PME en Pologne. Il a publié : « Promoting Small Business in the Post-communist Countries » dans Armand Clesse et Rudolh Tokés (éd.); The Economic and Social Imperatives of the Future Europe, Luxembourg, 1991 ; « New Opportunities for Entrepreneurs? » dans Krzysztof Kaczynski et Elzbieta Kawecka-Wyrzykowska (éd.) ; Re-integration of Poland into the West European Economy, Varsovie, 1992 et Maciej H. Grawboski et Przemyskaw Kulawczud (éd.); Small and Medium-size Enterprises in Poland Analysis and Policy Recommendation, Gdansk, 1992. Adresse : Institut pour l'économie de marché de Gdansk, 80-866 Gdansk, Gdynskich Kosynierow 11, Pologne. 


\begin{abstract}
This analysis shows how SMBs developed in Poland through the years 1980 to 1990 and how this development was affected by changes in the Polish economy, by the principal macroeconomic policies encouraged at the time and their impact on the growth of SMBs.

The author begins by highlighting the legal changes that made possible the rapid growth of incorporated SMBs as well as economic changes which took place toward the end of the 1980s. He then presents the basic principals which influenced these changes (the elements and results of the Balcerowicz plan), described the behaviour of private entreprises in relation to the changes and show hos the accelerated growth of SMBs came about in the second half of the 1990s.

In conclusion the author describes the impact of Polish macro-economic policies on the development of SMBs : the taxation system lopposed to the development of SMBs), high bank rates, deliberate inflation, and the failure of both taxation and fiscal policies.
\end{abstract}

\title{
RESUMEN
}

Este análisis muestra como se efectúa el desarrollo de las PME en Polonia entre los años 1980 y 1990 a traves los cambios ocuridos en la economía poolonesa, las principales políticas macroeconomicas efectuadas y su impacto sobre el desarrollo de la PME.

Primeramente, el autor enumera los cambios legales que han favorecido el crecimiento rápido de las PME incorporadas asi como los cambios económicos ocuridos a fines de la década 1980. En segundo lugar, el autor presenta los principios de base que han guiado éstos cambios (los elementos y los resultados del plan Balcerowicz), describe el comportamiento de las empressas privadas respecto a estos cambios y muestra como es sobrevenido la aceleracion del desarrollo de las PME en la seguda mitad de 1990.

Enfin, el autor describe el impacto de las políticas macro-economicas polonesas sobre el desarrollo de las PME : el fisco (defavorable al desarrollo de las PME, los elevados porcentages de interes bancarios, la inflacion provocada asi mismo el fracasso de la politica de impeuestos y de la politica fiscal. 


\section{Introduction}

Au début des années 80 , le secteur privé de l'économie polonaise ne comprenait que des entreprises non incorporées, la plupart artisanales. Elles relevaient à peu près exclusivement de propriétaires uniques, avec parfois quelques partenaires. À cause de la loi sur la nationalisation, les entreprises privées ne pouvaient embaucher plus de 50 travailleurs. La taille moyenne de ces entreprises était de moins de 2 employés (voir tableau 1). Leurs activités portaient surtout sur les services aux ménages et les services au commerce de détail et aux firmes manufacturières.

\section{TABLEAU 1}

Les PME du secteur privé en Pologne dans les années 1989 et 1990

Emploi

\begin{tabular}{|l|c|r|r|r|r|}
\hline & 1980 & 1985 & 1988 & 1989 & \multicolumn{1}{c|}{1990} \\
\hline 1. Non incorporées * & 601200 & 869300 & 1100000 & 1430000 & 1915500 \\
\hline $\begin{array}{l}\text { 2. Entreprises } \\
\text { étrangères» }\end{array}$ & 1560 & 54000 & 82000 & 100200 & 83230 \\
\hline $\begin{array}{l}\text { 3. Firmes } \\
\text { incorporées ** }\end{array}$ & n.a. & n.a. & n.a. & 134700 & 306240 \\
\hline $\begin{array}{l}\text { 4. Coentreprises } \\
\text { TOTAL }\end{array}$ & 602700 & 927700 & 1181000 & 1694400 & 2393040 \\
\hline
\end{tabular}

* Propriétaire unique et sociétés

** Compagnies à capital action et à responsabilités limitées 


\section{Nombre d'entreprises}

\begin{tabular}{|l|r|r|r|r|r|}
\hline & 1980 & 1985 & 1988 & 1989 & \multicolumn{1}{|c|}{1990} \\
\hline \begin{tabular}{l} 
1. Non incorporées * \\
\hline $\begin{array}{l}\text { 2. Entreprises } \\
\text { étrangères» }\end{array}$
\end{tabular} & 637200 & 418000 & 660000 & 814500 & 1135600 \\
\hline $\begin{array}{l}\text { 3. Firmes } \\
\text { incorporées ** }\end{array}$ & n.a. & n.a. & 1275 & 11901 & 29839 \\
\hline 4. Coentreprises & n.a. & n.a. & 3279 & 1761 \\
\hline
\end{tabular}

* Propriétaire unique et sociétés

** Compagnies à capital action et à responsabilités limitées

TABLEAU 2

Emplois en Pologne par secteurs, en décembre 1988*

\begin{tabular}{|c|c|c|c|c|}
\hline \multirow{3}{*}{ Secteur étatique } & \multirow{3}{*}{ Total } & \multicolumn{3}{|c|}{ Secteur privé } \\
\hline & & Agricole & \multicolumn{2}{|c|}{ Non agricole } \\
\hline & & & Propriétaires & Employés \\
\hline $\begin{array}{r}\text { En pourcentage } \\
71,7\end{array}$ & 28,3 & 21,9 & 3,0 & 3,4 \\
\hline $\begin{array}{l}\text { En milliers } \\
\qquad 13,230\end{array}$ & 5,220 & 4,040 & 550 & 630 \\
\hline
\end{tabular}

* Selon le dernier recensement 
La part des petites entreprises d'État (moins de 50 employés) dans les PME était marginale pendant toute la dernière décennie et employait quelques 5000 travailleurs en 1980 (en incluant les employés des petites coopératives).

Le secteur de la PME comprenait aussi les coentreprises « étrangères », c'est-à-dire les PME dirigées par des non résidents polonais (répertoriées dans la catégorie des entreprises non incorporées). Il n'y en avait que 46 en 1980, employant 1500 personnes. Ces entreprises pouvaient être enregistrées selon le réglementation de 1976 relevant du Conseil des ministres ; une permission spéciale était alors nécessaire. En 1989, 727 entrepriscs « étrangères » polonaises employaient 100000 personnes.

\section{Le développement des PME dans les années 80}

\subsection{Les changements légaux et le développement des PME}

En 1980, il n'y avait aucune entreprise privée incorporée ou coopérative indépendante. Tout le secteur coopératif était sous le contrôle de l'État : ces autorités avaient décidé d'équiper les coopératives pour les produits semi-finis et le matériel de base. Cela était crucial dans une économie de rareté. L'État conservait directement ou indirectement une influence sur leur gestion.

Au début de ces années, le secteur de la PME non agricole comprenait environ 357000 entreprises incorporées et employait 653000 personnes. En 1982, la nouvelle loi sur les coopératives fut introduite, et, en conséquence, ce secteur s'est développé spécialement dans les grandes villes. La plupart des coopératives rendaient des services aux entreprises d'État, mais une autorisation venant du bureau central était encore nécessaire pour l'établissement d'une nouvelle coopérative. Cette autorisation était habituellement difficile à obtenir. Les nouvelles coopératives étaient cependant entièrement autonomes.

Il est très difficile d'estimer l'évolution de ce secteur durant ces années parce que le système statistique ne faisait à l'époque aucune distinction entre les « nouvelles » coopératives et les anciennes. En 1980 et 1981 , on peut penser que beaucoup de membres d'ex-Solidarité, qui ne trouvaient plus d'emploi dans les entreprises d'État, furent directement impliqués dans la création d'un grand nombre de coopératives. Les « nouvelles » coopératives étaient plus petites (avec moins de 100 employés) que les anciennes. Toutefois, l'emploi dans celles-ci a augmenté entre 1980 et 1989 plus de trois fois pour atteindre 32500 personnes. On peut estimer que cette augmentation provenait avant tout de ces « nouvelles » coopératives. 
En avril 1988, la nouvelle loi sur les investissements étrangers fut introduite. À partir de juillet, les coentreprises «étrangères » purent s'enregistrer et en 1988 une autorisation fut donnée à 52 entreprises d'accepter une participation étrangère. Puis en 1989, une telle autorisation fut accordée à 866 entreprises, la plupart petites. La part de ces compagnies avec leur capital de départ de 100000 \$ était de $60 \%$ de toutes les co-entreprises conjointes. En 1989, le nombre d'employés de toutes ces coentreprises « étrangères » s'élevait à 29900 , mais seulement 419 d'entre elles étaient en activité et payaient des impôts.

Le plus important changement légal pour les PME fut introduit en janvier 1989. La nouvelle loi pour l'activité économique nécessitait beaucoup de licences et d'inscriptions (enregistrements) pour les entreprises privées. Elle accordait une importance spéciale au développement des entreprises ; à la fin de 1988, il y avait 659600 de ces entreprises qui employaient 1100000 personnes, alors qu'un an plus tard, ces données étaient respectivement de 814000 et 1430000 .

En considérant toutes les catégories d'entreprises privées, c'est-à-dire les firmes non incorporées, les entreprises « étrangères », les entreprises incorporées, on comptait 1780000 employés dans le secteur privé en 1989. Ainsi, le secteur de la PME polonaise repose presque entièrement sur la petite et très petite entreprise.

\subsection{La croissance rapide des PME incorporées}

Le changement dans la législation des pratiques légales a permis l'enregistrement de la première compagnie privée à responsabilité limitće en 1985. Cette loi n'était pas nouvelle, mais provenait des changements dans les lois existantes. Depuis 1985, les compagnies à responsabilité limitée furent enregistrées par la cour selon le Code du Commerce de 1934. Les compagnies incorporées étaient de plus grande taille par comparaison aux compagnies non incorporées et en 1989, elles employaient en moyenne 14 personnes. Elles se divisaient en compagnies privées et en compagnies d'État (la majorité des parts appartenant aux entreprises publiques). Jusqu'à à la fin de 1988, il y avait 5470 compagnies privées à responsabilité limitée en Pologne, avec 21824 actionnaires privés ; ces firmes employaient environ 65000 personnes. Un an plus tard, à la fin de 1989, elles étaient 11807 et employaient 134714 personnes (par comparaison, il y avait 4293 entreprises d'État incorporées). Le taux de roulement des entreprises privées incorporées dans le secteur privé non agricole était de $18 \%$.

Dans les années 1980, il y eut donc beaucoup de changement dans les caractéristiques du secteur privé polonais, avec la croissance rapide du nombre des entreprises incorporées, favorisée par la réactivation du Code du Commerce 
de 1934. Il fournissait des règles faciles et transparentes pour l'établissement des compagnies à responsabilité limitée et des co-compagnies. Plusieurs raisons expliquent cette création des entreprises sous forme de corporations :

1. Le lancement était facile. Les corporations enregistrées étaient en meilleure position pour obtenir des licences et autorisations administratives nécessaires.

2. La corporation était libre d'arranger ses contacts d'affaires, de faire une demande d'équipement, de choisir ses politiques financières, de recruter des travailleurs (ou faire une demande pour engager plus d'cmployés dans le secteur manufacturier). Par exemple, les possibilités d'affaires et de relations avec les firmes publiques étaient beaucoup plus limitées pour les entreprises non incorporées ;

3. Par définition, la corporation offrait les avantages d'une compagnie à responsabilité limitée. vantes :

Les nouvelles corporations privées présentaicnt les caractéristiques sui-

a) Le capital propre de démarrage était très faible et diminuait même. Selon une recherche réalisée en juin 1987, le capital propre moyen de départ dans la région de Gdansk est passé de 1,29 millions de zlotys en 1985 à 0,85 million en 1986 et à 0,74 million en 1987 ( 1 million de zlotys valait à ce moment entre 600 et $1500 \$$ US selon le taux du marché noir).

b) Plusieurs corporations privées n'avaient pas de capital réel. Cela signifie que le capital propre n'était pas le facteur le plus important pour mettre en marche une entreprise.

c) Le facteur critique pour débuter avec succès unc opération était d'établir des relations avec les grandes entreprises publiques ou les autorités locales, de trouver des contrats et d'offrir des services ou des biens nécessaires. En fait, ce genre d'activité était bien connu par les cadres moyens des entreprises d'État ou des coopératives, et une bonne part des corporations privées a été créée justement pour ces derniers.

d) Les relations entre la propriété et le management étaient très étroites ; les dirigeants étaient habituellement choisis parmi les propriétaires. Les corporations peuvent donc être considérées comme un capital d'entreprise selon la réalité polonaise au même titre que les sociétés.

e) L'incorporation des entreprises était facilitée de diverses manières. En plus de la facilité des procédures pour l'obtention des licences, il y 
avait plusieurs avantages à mettre sur pied une entreprise sous forme de corporation : le niveau maximum d'impôt pour une corporation était de $65 \%$ dans la moitié des années 1980 et de $40 \%$ en 1989, alors que pour les entreprises non incorporées, il était de $85 \%$ ct $75 \%$ respectivement. Tous les contrats avec l'administration, le système légal et les entreprises étaient facilités pour les corporations.

f) Les fonds d'opération des corporations provenaient des fonds personnels des propriétaires, des amis, des emprunts auprès d'autres entreprises (leurs actifs étaient supérieurs aux dettes), des avances du secteur public (les autorités locales et les entreprises d'État) ou des délais dans les paiements pour l'impôt, la sécurité sociale et les dépenses à faire pour les employés. Le financement des prêts par les banques était plutôt rare et habituellement à court terme. Malheureusement, la méthode de collecte du capital propre auprès du public était encore plus rare.

g) Les corporations, en tant que petites entreprises capitalistes, étaient capables de travailler selon une culture managériale différente de celle des entreprises d'État ou des firmes non incorporćes. Elles étaient généralement dirigées par des jeunes possédant des qualités d'encadreurs et de communicateurs. La forme du management était simple et non hiérarchique. Ces entreprises n'ont pas hérité des mauvaises pratiques du secteur public, et elles possédaient l'ambition et l'enthousiasme des entreprises artisanales.

Toutes ces données du secteur privé des petites corporations en Pologne ont créé une situation tout à fait dynamique. Ainsi le sectcur privé non agricole polonais, en passant au système de marché en 1990, se trouvait en meilleure position que les autres pays d'Europe de l'Est. Avec plus de 34000 corporations privées à la fin du mois de mars 1991, ce secteur de la petite entreprise polonaise possède déjà une structure légale qui ne peut que faciliter sa croissance future.

\subsection{Les obstacles à la croissance}

Le développement du secteur privé est cependant limité de deux manières :

1. Directement, puisque certaines activités sont toujours limitées aux entreprises étatisées, étant donné qu'aucune autorisation n'est disponible pour le secteur privé.

2. Indirectement, puisque les associations d'artisans avaient toujours le droit de maintenir leur quasi monopole en autorisant ou non l'entrée des nouveaux venus, ce qui pouvait occasionner des dépenses, en termes de coûts 
(pot-de-vin) ou de temps pour avoir le droit d'exercer. Le secteur privé souffre, depuis les années 1980 de plusieurs types de restrictions légales. Il y a des freins à l'emploi, des restrictions au remplacement du personnel et à la disposition des profits, etc. L'obtention de l'autorisation d'exploiter une entreprise nécessitait de passer à travers de longues étapes pour enfin obtenir la décision administrative finale. Par exemple, pour exploiter une firme de services pour les ménages, un postulant devait obtenir des autorisations de la brigade d'incendie locale, du service de santé, de l'association locale des artisans, la confirmation du bureau fiscal local qu'il n'y avait pas de retards au paiement des taxes et d'impôts, la confirmation du registre central sur la criminalité que le demandeur n'avait pas commis de crime au cours des dix dernières années, et enfin, du comité des citoyens (le conseil du quartier). Cette procédure prenait de 3 à 6 mois puisque plusieurs vérifications et informations supplémentaires devaient être faites. C'était la seule manière pour une entreprise d'entrer en activité, mais à partie de 1986, lorsque la première entreprise incorporée a ćté créée, cela était possible sans autorisation administrative. Toutefois, l'inscription d'une compagnie incorporée à la cour régionale était nécessaire, ensuite, on pouvait appliquer pour plusieurs licences.

Le traitement fiscal des PME dans les années 80 différait selon le type d'affaires (incorporée ou non), la taille de l'entreprise (pour les périodes de paiement et le taux de taxation) et le passé fiscal de l'entreprise (en cas de désaccord dans le paiement des taxes selon la loi fiscale, les règles de taxation les plus sévères étaient appliquées). Dans les années 80 , les règles fiscales suivantes étaient en vigueur dans les PME du secteur privé :

a) Une taxe « en un seul montant » pour les petites entreprises employant moins de 5 employés. Cette taxe a évolué depuis 10 ans (en fonction de l'inflation). Cette forme fiscale était intéressante pour les petites entreprises et considérée comme modératrice (cette forme de taxation a été limitée depuis 1990 aux petites entreprises de services familiales et d'emploi autonome).

b) Une taxe « de base » pour les coopératives, pour les entreprises indépendantes membres des corporations d'artisans et employant plus de 5 personnes, avec des variations élevées selon les revenus. Les taux relatifs à cette taxe évoluaient selon le type d'entreprise et les fluctuations dans les profits variaient de 9 à $21 \%$ selon le cas. En effet, bon nombre d'entreprises essayaient de déclarer des prévisions de profit aussi réduites que possible et ce type d'entreprise taxée faiblement se retrouvait le plus souvent dans les services. Cette forme de taxation a été abolie en 1990. 
c) Les principes généraux de l'impôt variable évoluèrent vers une taxation progressive avec un taux de taxation marginal maximum de $85 \%$ en 1982, $75 \%$ en $1986,50 \%$ en 1988 et $40 \%$ en 1989.

La taxe variable était évaluée selon les ventes. Cette taxation était considérée dans les années 80 comme une sorte de pénalité pour les entreprises ayant un roulement très élevé. Les entreprises forcées de payer cette taxe selon ces règles cessaient en général leurs activités. Durant ce qu'on a appelé « la fin de siècle » du système communiste, cette forme de taxation n'était plus acceptable ; actuellement le taux de taxation maximal de $40 \%$ est la façon habituelle de taxer les petites entreprises. Malheureusement, le niveau de $40 \%$ est atteint très facilement par certaines entreprises et cette taxe représente ainsi un taux linéaire de $40 \%$ plutôt qu'une taxe progressive.

Le facteur le plus important pour le développement de la PME dans les années 80 fut l'abolition des contraintes provenant des changements légaux et de l'interprétation de la loi. La politique économique consistait alors à donner des facilités spéciales à certaines industries. Par exemple en 1986, l'exemption d'une taxe pour 10 ans était introduite pour les petits producteurs de matériaux de construction. Ces facilités particulières étaient habituellement liées à la pénurie de certains biens sur le marché et seules certaines petites entreprises pouvaient en profiter.

\subsection{Les changements à la fin des années 80 - de nouvelles règles dans un même environnement}

Dans les années 80, le développement des PME en Pologne fut la conséquence de la levée légale des barrières dans la politique économique. Le marché ne jouait pas encore un rôle important dans le processus. Dans une économie de rareté, il est plus difficile d'acheter que de vendre. Cette règle a affecté aussi le secteur privé. Cependant, d'autres habiletés étaient nécessaires pour les entrepreneurs avec la concurrence qui commençait à apparaître sur certains marchés. Par exemple, le marché informatique fut l'un des premiers marchés compétitifs, et ensuite la même situation est apparue sur le marché des fruits et légumes locaux. On retrouvait d'autres exemples de ce genre, mais qui continuaient à être des exceptions à la règle.

Plusieurs entrepreneurs ont tenu compte des réformes légales et ont changé leurs produits de même que les formes légales de leurs activités. Les premiers entrepreneurs qui ont mis sur pied des entreprises incorporées en 1985 et 1986 venaient des « nouvelles » coopératives ou des entreprises privées non incorporées. La même situation a prévalu pour les produits : lorsqu'il était possible 
d'entrer dans un nouveau champ en affaires, formellement limité, les anciens entrepreneurs l'ont fait plutôt que les nouveaux.

L'année 1989 a amené une nouvelle situation : si l'économie du marché n'existait pas, plusieurs barrières ont toutefois été levées. L'hyperinflation fut un nouveau facteur dans l'économie polonaise. Néanmoins, cette année fut propice au développement du secteur privé et les entrepreneurs ont réagi rapidement et se sont bien adaptés à la nouvelle situation.

Pendant les années 80 , le secteur privé polonais s'est accru assez rapidement, mais la part de ce secteur est demeurée faible dans l'économie. La politique économique laissait une grande place aux nouvelles affaires. De nouvelles formes d'entreprises (entreprises incorporées, coentreprises et entreprises « étrangères ») ont été créées. Celles-ci étaient généralement plus grandes et mieux équipées que les entreprises traditionnelles non incorporées. La part du commerce extérieur, l'emploi et l'investissement étaient supérieurs à la moyenne dans celles-ci. Les propriétaires uniques et les sociétés ont en général utilisé une technologie dépassée et se sont faiblement financés à partir des banques.

\section{Le changement économique des années 90}

\subsection{Les principes de base}

En 1990, le plan Balcerowicz pour l'économie polonaise touchait les éléments suivants :

1. Un taux de change stable et une convertibilité locale du zloty.

2. La suspension de la plupart des subsides aux entreprises d'État.

3. Un commerce extérieur dérégularisé.

4. Un marché libre pour la plupart des produits.

5. Des droits douaniers faibles.

6. Des salaires gelés dans les entreprises d'État.

Les résultats les plus importants furent :

1. Une diminution des taux d'inflation après trois mois seulement.

2. Une diminution des taux d'intérêt.

3. Un accès relativement facile à l'importation des biens . 
4. Une forte concurrence sur la plupart des marchés.

5. L'affaiblissement du pouvoir d'achat des ménages.

La situation de 90 était nouvelle pour l'économie polonaise, non seulement pour les entreprises d'État mais également pour la plupart des entreprises privées. Cependant, le processus d'adaptation des entreprises privées aux nouvelles conditions se déroulait beaucoup mieux que dans les entreprises publiques. Les PME privées ont changé de produits, ont recherché de nouveaux créneaux sur le marché et ont essayé d'augmenter les exportations lorsque la demande nationale n'était plus suffisante.

\subsection{Le profil du comportement des entreprises privées}

Pendant les trois premiers mois de 90 , les entreprises privées ont eu à prendre des décisions sur la façon de gérer les nouvelles opportunités et les obstacles qui apparaissaient sur le marché. L'inflation était trop élevée pour que l'investissement puisse passer par le crédit bancaire. Les biens importés concurrençaient déjà plusieurs productions nationales et ainsi un coup frein fut donné à la demande des ménages et du secteur d'État. La réaction à cette situation se fit de différentes façons, soit :

1. le passage d'une production à une autre ou le recours à des pratiques commerciales incluant l'import et l'export, améliorant ainsi la profitabilité des entreprises ;

2. l'ouverture de leur propre système de distribution afin de minimiser le prix des produits ;

3. l'amélioration de la qualité des produits et des conditions de distribution ;

4. l'amélioration de l'image du produit par le marketing et la publicité ;

5. la diminution de la production, du niveau de salaire réel et, particulièrement, le passage à l'économie de marché noir afin d'éviter la taxation ;

6. la suspension des activités formelles pour passer à l'économie de marché noir ;

7. ou l'abandon des affaires.

Les entreprises qui ont réagi positivement à la nouvelle situation $\left(1^{\mathrm{er}}, 2^{\mathrm{e}}\right.$, $3^{e}$ et $4^{e}$ cas) ont amélioré en général leur situation sur le marché. Ce n'était pas difficile de le faire en particulier sur le marché de produits à travail intensif, puisque la concurrence sur ce marché n'était pas très grande, ni dans les biens 
provenant des entreprises d'État ni dans les produits importés. En plus, la « mentalité de rechercher les bas prix » était et reste forte dans les ménages et les entreprises en Pologne.

En juin 1990, la part de l'activité économique dans le commerce a été renforcée par des exemptions de taxes pour les petits commerçants, leur facilitant l'accès aux stratégies appropriées.

La privatisation des magasins a été faite de façon formelle par l'État et chez les entreprises municipales et a grandement facilité la distribution. La première vente aux enchères a eu lieu au printemps 1990.En même temps, les propriétaires privés ont acquis les droits de propriété de leurs équipements (mais non de leur bâtiment). Ils ont aussi pu louer ou créer leurs propres entreprises.Mais formellement, ils n'étaient pas libres de louer leurs équipements et à n'importe quel prix.

\subsection{L'accélération du développement des PME dans la seconde moitié de 90}

À la fin du printemps 1990, lorsque le taux d'inflation baissa, l'investissement a pu à nouveau être financé par des crédits bancaires. L'industrie bancaire polonaise à majorité contrôlée par l'État ne constituait pas un partenaire approprié pour les entreprises privées; néanmoins, le montant des crédits pour les PME a augmenté. La situation est devenue toutefois dangereuse pour les entrepreneurs qui avaient déjà obtenu du crédit, quand le refinancement du taux de crédit a grimpé à $43 \%$ en octobre et à $72 \%$ en janvier 1991.

Pour plusieurs entreprises privées, il était très difficile de payer à nouveau ces intérêts bancaires ; ceux-ci ont été offerts pour des projets à court terme (fonds de roulement) plutôt que pour l'investissement. Aussi, la plupart des PME polonaises ne recoururent pas au crédit bancaire pour leur financement, utilisant plutôt leurs épargnes personnelles, les emprunts informcls et leurs bénéfices.

En 1990, le développement des petites entreprises accéléra les disparités en Pologne. L'est du pays resta loin derrière la Silésie, les grandes villes, Wielkopolska (dans la région de Poznan) et en général, l'ouest et le centre de la Pologne (voir le tableau 3). Dans les régions où il n'y a pas eu un développement significatif du secteur privé non agricole, on a pu constater officiellement le déclin de l'activité économique. 
TABleAu 3

Changements d'emplois par secteurs et par régions

(8 sur 49) en 1990 (en milliers)

\begin{tabular}{|l|c|c|c|c|}
\hline & $\begin{array}{c}\text { Profit } \\
\text { des employés } \\
\text { du secteur } \\
\text { privé } \\
\text { en \%* }\end{array}$ & $\begin{array}{c}\text { Pertes } \\
\text { d'emplois } \\
\text { dans le secteur } \\
\text { étatique } \\
\text { A }\end{array}$ & $\begin{array}{c}\text { Pertes } \\
\text { d'emplois } \\
\text { dans le secteur } \\
\text { privé } \\
\text { B }\end{array}$ & $\begin{array}{c}\text { Ratio } \\
\text { en \% }\end{array}$ \\
\hline POLOGNE & 28,3 & 1970,5 & 521,8 & 26,5 \\
\hline Warszawa & 18,3 & 135,7 & 48,1 & 35,4 \\
Gdansk & 17,0 & 83,5 & 28,2 & 33,8 \\
Lodz & 17,7 & 66,7 & 19,4 & 29,1 \\
Wroclaw & 39,5 & 57,6 & 26,6 & 46,2 \\
Krosno ** & 37,5 & 24,8 & 2,2 & 8,9 \\
Lomza ** & 56,8 & 12,3 & 0,1 & 0,8 \\
Przemysl ** & 42,4 & 18,6 & 1,8 & 9,7 \\
Suwalki ** & 33,6 & 21,5 & 1,6 & 7,4 \\
\hline
\end{tabular}

* Incluant l'agriculture, 1988

** Régions dans l'est de la Pologne

Les PME polonaises se sont développées de façon disproportionnée selon les secteurs. Par exemple, on a vu une croissance significative des entreprises commerciales, alors que déclinaient les services et le secteur manufacturier. Plusieurs nouvelles entreprises commerciales se sont établies et bon nombre d'entreprises manufacturières sont passées au commerce. Cette restructuration des PME dans l'industrie polonaise présente trois caractéristiques.

D'abord, les différences dans les profits entre les diverses productions ont beaucoup augmenté au cours de la dernière année. Deuxièmement, plusieurs productions spécifiques ont pu facilement être exportées sur les marchés étrangers. Enfin, les actifs dans les petites entreprises polonaises étaient faibles, ce qui a facilité les changements d'activité.

Les règlements sur les échanges ou reliés à la politique économique (comme le taux d'intérêt, les taxes, les contingentements à l'exportation) ont 
modéré le désir d'investir, puisque les décisions administratives pouvaient entraîner un accroissement ou une baisse du taux de profit.

La croissance la plus élevée parmi les différentes catégories de PME se trouvait dans les entreprises conjointes et les compagnies incorporées. Ces entreprises étaient généralement de petite taille et complétaient de façon significative la production des entreprises de taille moyenne (voir tableau 1). Les monopoles ou quasi monopoles d'État n'ont pu défendre leurs positions et ont perdu leur part de marché en faveur des petites entreprises privées. Les tendances dans les PME en 1990 (tel le déclin des PME dans l'Est de la Pologne ou le développement rapide des entreprises commerciales) se sont maintenues en 1991. En avril de cette année, la part du secteur privé du secteur manufacturier a atteint $17,5 \%$ (en 1989, environ $10 \%$ ), celle de la construction, $41 \%$, celle de l'importation, $36,4 \%$, et enfin, celle de l'exportation, $12,5 \%$.

\section{Les politiques macro-économiques et leur impact sur le développement des PME}

\subsection{La fiscalité opposée au développement des PME : un phénomène typiquement polonais}

Il est évident que toute création d'entreprises, spécialement celle des PME, nécessite des ressources financières. Les PME sont très sensibles aux décisions d'une politique économique qui change trop souvent, en particulier à cause des ressources financières trop faibles de ces entreprises. Compte tenu des divers éléments déjà mentionnés, il faut que l'administration adopte diverses mesures pour soutenir rapidement les affaires. Toutefois, certaines de ces facilités, dans des conditions données, peuvent parfois avoir des effets pervers sur toute l'économie.

C'est une situation de ce genre qui est apparue en 1991 en Pologne et a causé une crise budgétaire sérieuse. Cette crise rendit impossible l'utilisation des ressources gouvernementales pour la promotion des PME. La principale cause de cette situation provenait de la mesure introduite l'année précédente. En effet, en mai 1990, le gouvernement a permis de reporter le paiement des impôts de un à trois ans pour les entreprises privées nouvellement établies, principalement dans le secteur commercial et en partie dans les secteurs de services. Cette mesure a été implantée pour accélérer le processus de privatisation du commerce.

Cet instrument a été un succès pour la privatisation, mais a limité énormément les profits des commerces à cause de leur nombre trop élevé. Pour com- 
penser les pertes, le gouvernement a essayé d'accroître les droits de douanes et la taxe sur les ventes des biens importés, mais ceci n'a fait que limiter les importations et n'a pas donné les résultats positifs escomptés pour l'État. Il est très important de souligner que le commerce est le seul secteur de l'ćconomie nationale qui soit relativement en santé. Le gaspillage des profits de commerce a obligé à accélérer la politique de diminution des subsides pour les biens comme l'électricité, le charbon, le gaz, etc. L'inflation qui a suivi cette action a créé une tension entre le ministre des Finances et la Banque Nationale. Finalement, le niveau des taux d'intérêt de la Banque Centrale a graduellement grimpé à $36 \%$ en octobre 1990 et à $72 \%$ en mars 1991. Cette croissance des taux d'intérêt a bloqué l'investissement dans le secteur privé et plusieurs entreprises manufacturières privées ont dû réduire leur production. La conclusion générale qui s'en dégage est qu'une politique fiscale qui ne tient pas compte de l'impact sur les profits finit par se couper l'herbe sous le pied et limite les ressources budgétaires pour financer les programmes de promotion des PME.

\subsection{Les taux d'intérêt bancaires et l'inflation provoquée}

L'arme la plus impressionnante de la politique macro-économique de la Pologne était de maintenir un taux d'intérêt bancaire positif par rapport à l'inflation. Or, l'inflation polonaise de 1991 peut être vue comme une inflation provoquée par le gouvernement. Toutes les vagues d'inflation de cette année ont découlé des décisions gouvernementales, comme la diminution du taux d'échange de la monnaie polonaise, l'augmentation des droits de douane et des taxes à l'importation ou l'augmentation du prix des biens fixés par le gouvernement. Ces décisions, bien que logiques et nécessaires, ont accéléré l'inflation. L'augmentation de l'inflation a entraîné l'augmentation du taux d'escompte de la Banque Centrale et finalement le blocage de l'activité économique. Tant que les taux d'intérêt variaient entre $60 \%$ et $100 \%$ par année, il n'était plus possible de financer l'activité économique pour des investissements à long terme (comme la construction immobilière et navale, et bon nombre d'activités manufacturières) qui nécessitent le recours à des emprunts bancaires. Il est aussi important de souligner que le taux de change était relié à un groupe de monnaies. La seule branche de l'économie qui pouvait être financée par les banques était le commerce où les cycles de retour sur investissement pouvaient se limiter à 20 ou 30 jours.

Les entreprises qui, en 1990, ont emprunté pour financer leurs investissements en souffrent actuellement. À cause des taux d'intérêt de $60 \%$ à $100 \%$ d'alors, elles paient plus en intérêts pour des anciens prêts que pour des nouveaux. L'investissement à moyen terme basé sur des emprunts bancaires demcurait très difficile et, à long terme, impossible. La seule manière de con- 
trer l'augmentation des taux d'intérêt était d'essayer d'obtenir du crédit en monnaies étrangères. Ces crédits étaient cependant distribués par les banques et il était logique de s'attendre à ce que cette source devienne rapidement insuffisante et crée " des listes d'attente », entraîne des comportements douteux, et finalement la corruption. Il semble surprenant que le ministre des Finances ait autorisé des prêts pour des marchés étrangers parallèles avec des taux d'intérêt significativement faibles, alors que la politique locale était extrêmement restrictive.

\subsection{La politique de taxation et la politique fiscale}

L'échec de la politique fiscale (le paiement des taxes) est le résultat le plus visible d'une politique inappropriée de taxation. La division du secteur privé en deux parties, la première taxée et la seconde non taxée, a créé une situation dans laquelle une bonne partie de l'impôt restait impayé.

Il existait beaucoup de mécanismes pour éviter de payer les taxes sur les revenus des entreprises. Deux d'entre elles étaient relativement efficaces. La première avait trait au recours à des prix transférables. Par exemple, les prix dans les compagnies manufacturières étaient généralement réduits afin de payer un minimum d'impôts sur le revenu ;les biens étaient vendus aux firmes commerciales qui coopéraient et qui haussaient ensuite les prix autant que possible. Comme les revenus en commerce n'étaient pas taxés, l'État ne percevait plus de taxes. Il n'existait aucun moyen pour contrer ces opérations et empêcher de tels mécanismes. Le second exemple est la manipulation des coûts. Cette manipulation peut être définie comme l'augmentation de certains coûts afin de présenter une mince différence entre les prix de ventes ct les coûts. Ces coûts " manipulés » sont malhonnêtes, mais il n'existe aucune façon de les éviter dans la législation actuelle. Par ailleurs, le manque de contrôle fiscal efficace a fait en sorte que les affaires « légales » ne sont pas très loin « des affaires au noir ». Tout cela a limité la possibilité d'implanter des mesures avantageuses comme le crédit sur les taxes d'investissement ou l'assistance au capital propre, etc.

\subsection{Conclusions sur la politique macro-économique et ses impacts sur le développement de la PME}

À notre avis, sur la politique macro-économique on peut arriver aux conclusions suivantes :

1. La situation du budget actuel commande de mettre de l'ordre dans les politiques fiscales. Cet ordre devra être basé sur le principe que toutes les entreprises doivent payer les taxes, ce qui devrait améliorer la situation. 
2. Les taux d'intérêts élevés de la Banque Centrale, nuisibles à l'économie, devront être réduits afin de promouvoir l'activité économique.

3. Pour améliorer le contrôle du budget de l'État, les programmes d'assistance financière pour les PME devront être activés. Ces programmes devront privilégier les PME mentionnées plus haut et devront aider à la formation du capital à moyen et à long terme pour soutenir les investissements et le démarrage d'entreprise.

4. Le rôle exagéré du ministre des Finances devra être réduit et les fonctions de régulation monétaire devront passer à une Banque Centrale indépendante.

5. L'accès au marché financier pour les PME devra être élargi. Ceci suppose la suppression des procédures restrictives d'autorisation de nouvelles banques et autres institutions financières. La concurrence sur le marché financier est la meilleure voie pour améliorer l'accès des PME au financement bancaire. 